\title{
Einleitung zum Themenheft Pragmatik der Genuszuweisung*
}

\author{
Helen Christen (Freiburg i. Ü.), Gerda Baumgartner (Freiburg i. Ü.), Melanie Bösiger \\ (Freiburg i. Ü.), Simone Busley (Mainz), Julia Fritzinger (Mainz), Peter Gilles (Luxem- \\ burg), Damaris Nübling (Mainz), Martina Schläpfer (Freiburg i. Ü.)
}

Man könnte einwenden, dass der Titel des vorliegenden Sammelbandes - „Pragmatik der Genusverwendung" - einen Widerspruch in sich beinhalte: Schließlich gilt doch als grammatiktheoretische Grundkonstante, dass die Kategorie des Genus Substantiven inhärent ist und fest mit bestimmten lexikalischen Kategorien assoziiert ist. Doch nicht zuletzt seit der Entwicklung von Corbetts ,Agreement Hierachy“ (cf. Corbett 1991, 2006) kristallisiert sich mehr und mehr heraus, dass die eigentlich feste grammatische Kategorie des Genus durchaus variabel erscheinen kann. Es sind insbesondere spezifische pragmatische Gebrauchskontexte und syntaktische Kongruenzrelationen, in denen für manche Sprachen und Varietäten eben abweichende Genusverwendungen zu beobachten sind, die unseres Wissens nach bis heute noch umfassender empirischer Dokumentation und einer systematischen Analyse harren. In einer frühen Studie zur Referenz auf weibliche Personen hat Christen (1998) gezeigt, dass die Kongruenz zwischen Controllern und Targets stark durch soziopragmatische Faktoren wie Vertrautheit, emotionale Nähe/Distanz oder Mitleid gesteuert sein kann. Es sind insbesondere alemannische und westmitteldeutsche Varietäten, in denen die auffälligen sog. ,Femineutra' vom Typ das Melanie, also Neutrumsverwendungen für weibliche Referenzpersonen, vorkommen. Die Beiträge dieses Bandes analysieren folglich größtenteils Genusverwendungen, die in bestimmten pragmatischen Kontexten vom inhärenten Genus des nominalen Controllers entweder abweichen oder variabel und mit bestimmten pragmatischen Wirkungen eingesetzt werden können.

Die Beiträge des Bandes sind in zwei Sektionen eingeteilt: Am Beginn des Bandes stehen Beiträge, die sich mit der Genusverwendung aus der Perspektive spezifischer lexikalischer oder konstruktioneller Einheiten wie Mädchen, Backfisch, 's Bachmanns Anna, unser Dietmar, Adressierungen in der 2. Person Singular oder der Pronominalisierung von Hunden beschäftigen (Beiträge von Hübner, Stocker, Schweden, Werth, Simon, Baumgartner et al.). In der zweiten Sektion werden dann regionalspezifische Genusverwendungen im Westmitteldeutschen und Alemannischen diskutiert, wo sich u. a. die charakteristischen Femineutra in je unterschiedlichem Grad erhalten haben (Beiträge von Busley/Fritzinger, Dammel, Christen/Baumgartner, Bösiger, Zürrer).

Die Beiträge resultieren aus der Tagung ,Pragmatik der Genuszuweisung“, die vom 1. bis 2. Februar 2019 an der Universität Freiburg i. Ü. stattfand und Teil des trinationalen Projekts „Das

\footnotetext{
* Wir bedanken uns bei Elke Hentschel sehr herzlich für die Aufnahme dieses Themenhefts in Linguistik online sowie bei allen Reviewerinnen und Reviewern für ihr kompetentes und konstruktives Feedback zu den eingereichten Beiträgen. Unser Dank gilt ausserdem Ingrid Hove für die sorgfältige Durchsicht der englischen Abstracts und Celine Muhr für das gewissenhafte Korrekturlesen.
}

Linguistik online 107, 2/21 - http://dx.doi.org/10.13092/lo.107.7681

CC by 3.0 
Anna und ihr Hund - Weibliche Rufnamen im Neutrum. Soziopragmatische vs. semantische Genuszuweisung in Dialekten des Deutschen und Luxemburgischen“"war, das von 2015 bis 2019 an den Universitäten Mainz, Freiburg i. Ü. und Luxemburg durchgeführt wurde.

\section{Überblick über die Beiträge}

In ihrem Beitrag „Genus und Sexus im Konflikt. Kongruenzformen hybrider Nomina im Sprachproduktionsprozess" widmet sich Julia Hübner dem Kongruenzverhalten von Mädchen. Dieses Lexem weist als sogenanntes ,Hybrid Noun“ eine inkonsistente Genuszuweisung auf, die sich darin äußert, dass anaphorische Elemente sowohl im Femininum als auch im Neutrum auftreten können (z. B. das Mädchen - sieles). Empirisch liefert Hübner Evidenz gegen die Annahme der traditionellen, substantivzentrierten Genusforschung, dass diese Hybridität durch miteinander konkurrierende grammatische und semantische Merkmale des Nomens zu erklären sei: Das Diminutivsuffiv -chen löse das Neutrum aus, die lexeminhärente Sexusinformation [+weiblich] das Femininum. Dem widerspricht bereits die Studie von Braun/Haig (2010), die derartige Genuskonflikte wortextern verorten und auf Eigenschaften der Referentin von Mädchen zurückführen. Sie können nachweisen, dass die Wahrscheinlichkeit femininer Kongruenz bei Mädchen, die älter als zwölf Jahre alt sind, wahrscheinlicher ist als bei jüngeren, was mit der Geschlechtsreife der Referentin begründet wird. Hübner, die sich in ihrer Fragebogenstudie auf das Genus von Relativpronomen fokussiert, greift diesen Ansatz auf. Sie kann dabei belegen, dass es nicht nur die Geschlechtsreife ist, die die Genuswahl determinieren kann, sondern darüber hinaus die Sexualisierung der Referentin: Wird Mädchen mit Referenz auf eine geschlechtsreife Frau in sexuelle Kontexte platziert, fällt der Femininumanteil signifikant höher aus als in neutralen Kontexten, er dominiert sogar den Neutrumanteil. Dies ist auch insofern bemerkenswert, als Relativpronomen von allen pronominalen Wortarten am stärksten zu formaler Kongruenz mit dem Bezugswort neigen. In nicht-sexuellen Kontexten sind die Femininumwerte in Hübners Studie außerdem bei Interaktionen des Mädchens mit einem Mann höher als bei solchen mit einer Frau. Damit identifiziert Hübner pragmatische Einflüsse auf die Genuszuweisung und zeigt so, dass eine rein lexikalische Verortung von Genus nicht haltbar ist.

Christa Stocker nimmt in ihrem Beitrag die weibliche Personenbezeichnung Backfisch zum Anlass, der Funktion von Genus-Sexus-Inkongruenzen im Inventar von historischen Bezeichnungen für junge Frauen nachzugehen. Die weibliche Personenbezeichnung Backfisch, die im Maskulinum steht, meint ein Mädchen am Übergang zur jungen Frau, das gewisse Charakterzüge aufweist, die üblicherweise eher dem männlichen Geschlecht zugeschrieben werden. Vor dem Hintergrund anderer Personenbezeichnungen mit Genus-Sexus-Inkongruenz geht die Autorin der Frage nach, inwiefern mit dem divergenten Genus eine pejorative Bedeutung einhergeht und die Genuszuweisung somit Mittel ist, eine soziale Normverletzung sprachlich zu markieren. Der Weg vom Kind zur (verheirateten) Frau im Bürgertum wird an frequenten kategoriengebundenen Personenbezeichnungen in historischen Mädchenbüchern und -zeitschriften aus dem 19. Jahrhundert nachgezeichnet. Dabei wird deutlich, dass Personen des weiblichen Geschlechts auf ihrem Weg zur Ehefrau, die mit dem Femininum assoziiert ist, offensichtlich das gesamte Genussystem durchschreiten.

In ihrem Beitrag „S (Bachmanns) Anna. Die Rolle der Herkunftsfamilie bei Referenz und Namengebung in dörflichen Kommunikationsgemeinschaften“ zeigt Theresa Schweden anhand 
von Sprachdaten aus verschiedenen bundesdeutschen Orten, wie sich patriarchale Strukturen in der dialektalen Personenreferenz verfestigt haben. Den Entstehungshintergrund der von ihr untersuchten Haus- und Personennamen plausibilisiert Schweden sozialhistorisch mit der Abhängigkeit der Hausbewohner_innen vom männlichen Hausvorstand. Bis heute werden Häuser, deren Namen in dörflichen Kommunikationsgemeinschaften zur Lokalisierung und Identifizierung von Familien verwendet werden, in der Regel nach dem männlichen Hausvorstand benannt. Der Fokus des Beitrags liegt auf der zum Standarddeutschen inversen Serialisierung von Familienname und Rufname, die sich durch Variation bei der Genitivmarkierung am Familiennamen (stark, schwach, keine Markierung) und beim Artikelgebrauch (Genitivartikel, flektierbarer Artikel, kein Artikel) auszeichnet, z. B. (der/s) Schmidt-Ø/-en/-s Karl. Der vorangestellte Familienname bleibt auch nach einem Namenwechsel durch Heirat in der Regel derjenige des Vaters. Seine primäre Funktion liegt Schweden zufolge in der onymischen Differenzierung von Personen, die über den Verweis auf die Herkunftsfamilie gelingt. Die unterschiedlichen formalen Typen sind nicht nur areal distribuiert, sondern es variieren in manchen Orten verschiedene Typen paradigmatisch. Liegt letzteres vor, können insbesondere über den Artikelgebrauch soziale Differenzierungen (Alter, Geschlecht) vorgenommen werden. Schweden hebt den Genitivartikel $s$ besonders hervor, der bei jüngeren Sprecher_innen des rheinfränkischen Dialekts von Höringen konzeptuelle Überschneidungen mit Femineutra aufweist: Hier tritt er nicht nur bei der Referenz auf Kinder beider Geschlechter auf, sondern auch bei Bezug auf erwachsene, nahestehende Frauen, deren Familien in der Ortsgemeinschaft etabliert sind.

In seinem Beitrag „Grammatik, Höflichkeit und Gender in der Zweiten Person“ thematisiert Horst Simon verschiedene pragmatische Instantiierungen des Genus in Anredeformen. Dabei zeigt sich, dass die Anredepronomen der 2. Person und das damit verbundene ,Adressatengenus' immer auch mit Aspekten der Höflichkeit verbunden sind. Die Belege stammen aus einer Reihe von Sprachen, u. a. aus dem Japanischen, Arabischen und anderen semitischen Sprachen und aus dialektalen Varietäten des Deutschen und Italienischen.

Die Mensch-Tier-Grenze thematisieren Gerda Baumgartner, Simone Busley, Julia Fritzinger und Sara Martin in „Das Anna und ihr Hund - Weibliche Hundenamen im Neutrum?“. Hier gilt das Interesse dem Hund, genauer Hündin Frida, die in einem Video-Experiment ebenso zu sehen und spontan zu beschreiben war wie verschiedene, ebenfalls vornamentlich benannte Frauen und Mädchen. Während bei den weiblichen Personen das Genus ihrer Namen und Pronomina stark von Alter und soziopragmatischen Faktoren wie Status und Beziehung abhing, entfielen diese Faktoren für die Hündin. Auf diese wurde häufig mit maskulinen Appellativen wie Hund oder Möpp referiert. Anders im Luxemburgischen, wo das Neutrum die unmarkierte Referenz auf Mädchen und Frauen leistet und Hündinnen ebenso erfasst. (Schweizer-)Deutsche Dialekte schließen Hündinnen also von der üblichen Genuszuweisung aus. Wenn, dann werden sie eher im distanzierenden Femininum oder gar im ,Hundegenus Maskulinum‘ pronominalisiert, gemäß dem Appellativ Hund. Hündinnen können bzw. konnten früher sogar männliche Namen bekommen, heute sind es eher weibliche (mit femininem oder neutralem Artikel): die bzw. das Daisy. Doch die ihnen folgenden Pronomen kippen meist ins Maskulinum. Dies zeigt, dass ein Hund per se männlich assoziiert bzw. genderisiert ist: „Der weibliche Hund entspricht also konzeptuell eher einem maskulin klassifizierten Konzept „Hund“ als einem Konzept ,weiblicher Mensch", das mit Neutrum bzw. Femininum assoziiert ist.“" Die Mensch/Tier-Grenze wird somit genusgrammatisch gestützt.

ISSN 1615-3014 
Obwohl es in Dialekten sehr spezifische Verwendungsweisen der Konstruktion unser+Rufname gibt, hat diese bisher keinen Eingang in namengrammatische Beschreibungen gefunden. Alexander Werth kann mit seinem Beitrag „Soziopragmatik von unser bei Rufnamen im Westmitteldeutschen. Zum Gebrauch sprecherassoziierter Referenzausdrücke" nun aufzeigen, dass der Gebrauch des Possessivs unser keineswegs nur der Unterstützung der Referenzleistung dient, sondern ein soziopragmatisch gesteuerter Referenzausdruck ist, der die soziale Beziehung zwischen Sprecher_in und Referent_in interpretiert. Auf der Datenbasis von Interviews, die er an vier moselfränkischen Orten durchgeführt hat, kommt Werth zum Schluss, dass unser+Rufname in nähesprachlichen Kontexten verwendet wird und optional zum Zuge kommen kann, wenn biologische Verwandtschaft vorliegt oder wenn - im Falle von Schwiegerkindern - zumindest Sympathie vorhanden ist. Die besondere emotionale Verbundenheit, die mit der unser-Konstruktion ausgedrückt wird, und deren Gebrauchsspezifik zeigen unübersehbar Gemeinsamkeiten mit den moselfränkischen Femineutra. Während die Femineutra jedoch ausschließlich für die Referenz auf Frauen verwendet werden und die soziale Beziehung zwischen Sprecher_in und Referent_in ausdrücken, steht bei den unser-Konstruktionen die Markierung der biologischen Verwandtschaft im Vordergrund. Werth qualifiziert diesen Referenzausdruck insofern als „sprecherassoziiert“, als dieser - anders als etwa der onymische Artikel - eine Sprecherperspektive ins Spiel bringt, die die Übernahme von Verantwortlichkeit gegenüber der Referenzperson voraussetzt. Dieser Aspekt ist bei den Femineutra in weit geringerem Maße gegeben, was Alexander Werth ausblickende Forschungsdesiderata formulieren lässt, die darauf abzielen, variable Genuszuweisung und unser-Konstruktionen als Teil ganzer PersonenReferenzsysteme zu begreifen und künftig den Blick auf deren internes Zusammenspiel hinsichtlich Gebrauch und Funktion zu lenken.

Simone Busley und Julia Fritzinger gehen in „Neutrales Rufnamengenus zwischen Grammatik und Pragmatik" der Frage nach, inwiefern das Neutrum bei Bezug auf Frauen und Mädchen obligatorisch oder optional ist. Manche Dialekte haben Genus optionalisiert, für soziopragmatische Zwecke freigesetzt und funktionalisiert, womit Neutrum und Femininum in Opposition zueinander treten. Primär dient die Genuswahl der Anzeige der Beziehung der Sprechenden zum weiblichen Referenzobjekt. Hinzu kommen Eigenschaften der Referentin wie deren Alter, Familienrolle und Sozialstatus. Solche spezifischen syntagmatischen Genuskombinationen werden als Degrammatikalisierung bzw. Pragmatikalisierung der einstmals fest zugewiesenen, unverhandelbaren Genuskategorie gewertet, wobei ein neues (Mini-)Paradigma entsteht. Bei diesem Prozess wird auch das bisher rigide Kongruenzmuster gebrochen (die Ingrid - sie vs. es). Weitere Entwicklungen haben im Luxemburgischen und in angrenzenden deutschen Dialekten stattgefunden, wo aufgrund der hohen Neutrumfrequenzen $\mathrm{zu}$ - bekannten, daher mit Vornamen benannten - Frauen und Mädchen sich dieses Genus fest an jeglichen weiblichen Vornamen gebunden hat, so dass die Regel gilt: Weibliche Vornamen sind neutral und lösen auch entsprechende Kongruenz aus. Dabei haben sich sogar grammatisch neutrale, doch semantisch weibliche Sonderpronomen wie lux. hatt herausgebildet. Dieser Prozess wird als Regrammatikalisierung einstmaliger Genuspragmatik gefasst. Andere Dialekte sind (wieder) zum Femininum zurückgekehrt.

In ihrem Beitrag „Evaluative Wortbildung und Personenreferenz“ nimmt Antje Dammel das höchst produktive alemannische Wortbildungsmuster der $i$-Ableitungen in den Blick, das evaluative Personenbezeichnungen mit zumeist maskulinem Genus erzeugt. Der Sachverhalt, dass

ISSN 1615-3014 
$i$-Bildungen sowohl in der Onymik als auch in der Appellativik vorkommen, ist für Dammel der Schlüssel zum mutmaßlichen Entstehungsszenario des Derivationsmusters. Eine entscheidende Rolle scheinen dabei die hochfrequenten hypokoristischen männlichen Kurznamen auf $-i$ gespielt zu haben, deren maskulines Genus eine der Voraussetzungen für die diachron nachgeordneten maskulinen $i$-Personenbezeichnungen bildet. Als Brücke für den Übergang des onymischen Musters in die Appellativik setzt Dammel deonymisierte männliche Kurznamen wie Ludi (zu Ludwig) an, die entweder frei oder als Suffixoide (Suufludi m. ,Säufer') für negative Stereotypisierungen genutzt werden. Diesen Stereotypisierungen wendet sich Dammel im zweiten Teil ihres Beitrages zu und sie deckt anhand von Wörterbucheinträgen auf, welches normabweichende Verhalten in lexikalisierten Personenbezeichnungen sedimentiert ist.

Der Beitrag von Helen Christen und Gerda Baumgartner fokussiert auf die Dialekte der Deutschschweiz und geht der sog. „Diminutiv-Hypothese“ nach, derzufolge Diminutivsuffixe und das durch sie morphologisch ausgelöste neutrale Genus Ausgangspunkt des Neutrums nicht-diminuierter Rufnamen sind. Ihre Analysen, basierend auf Daten aus dem Forschungsprojekt „Das Anna und ihr Hund - Weibliche Rufnamen im Neutrum“, Todesanzeigen sowie v. a. belletristischer historischer Belege, bestätigen diese Hypothese. Die Autorinnen decken eine geschlechtsspezifische Asymmetrie bei der Diminution von Ruf- und Verwandtschaftsnamen auf, die sich insbesondere seit dem 19. Jahrhundert verfestigt und die $l i$-Diminutive sowie neutrales Genus auf Frauennamen beschränkt ( $d s$ Anneli, ds Mami), wohingegen Männernamen mit $i$-Suffix diminuiert werden und stets sexuskongruent maskulin sind (de Ruedi). Die vornehmliche Assoziation mit weiblichem Geschlecht führen sie darauf zurück, dass sich der durch die Diminution indizierte Intimitätsgrad sowie die mit dem Neutrum einhergehende Deagentivierung eher mit weiblichen als mit männlichen Genderkonzepten vereinbaren lässt. Ein Ausblick auf Suffixe, die zu einer geschlechtsinversen Genuszuweisung (sog. ,gender reversal“) führen, leitet die Autorinnen zu der abschließenden These, dass die Suffixe bloße Mittel zum Zweck der Herstellung ikonischer, d. h. Geschlechtsrollenübertretungen markierender GenusSexus-Diskordanzen sind.

Der Fokus im Beitrag von Melanie Bösiger liegt auf dem onymischen Artikel und der Frage nach dessen grammatischer Funktion im Schweizerdeutschen, wo der Artikel mit der Ausnahme weniger höchstalemannischer Dialekte grundsätzlich als obligatorisch gilt. An ausgewählten Umfragedaten aus der gesamten Deutschschweiz lässt sich die Annahme erhärten, dass im aktuellen Sprachgebrauch nicht mehr die Anzeige von Kasus, sondern vielmehr die Genusmarkierung zur primären Funktion des Artikels geworden ist. Interessanterweise ist dieser Wandel gerade dort zu beobachten, wo der onymische Artikel nicht obligatorisch ist und im Gebrauch von weiblichen und ggf. männlichen Rufnamen mehrere Genera möglich sind: Der onymische Artikel scheint vor allem dann eingesetzt zu werden, wenn Genus und Sexus voneinander abweichen können, also bei männlichen Rufnamen im Neutrum wie ds Chrischti (zu Christian) oder weiblichen Rufnamen mit variablem Genus (d Anna/ds Anna). Als aufschlussreich für die Analyse erweisen sich insbesondere Possessivkonstruktionen mit Genitiv, die auffällige Mischformen zutage fördern.

Besonders weit gediehene Entwicklungen beschreibt Peter Zürrer in „Genus-Zuweisung bei der Pronominalisierung von Personen in den Südwalser Dialekten“. In diesen Sprachinseldialekten auf italienischem Gebiet hat sich das nominale, doch primär das pronominale Neutrum 
bei soziopragmatischem Näheverhältnis, bei Empathie und bei symmetrischer Beziehung zwischen Sprecher_in und Referent_in zum unmarkierten Genus jeglichen Personenbezugs entwickelt. Dieses gilt somit für beide Geschlechter, unabhängig davon, ob deren Namen oder Appellative diminuiert sind oder nicht ( $d$ s sielig Edmond, der verstorbene Edmond'). Auch die dort geltende verbale und prädikative Genuskongruenz folgt dem Neutrum und grammatikalisiert beim Verb als finales $-s$ zu einer allgemeinen Personalendung (vgl. dar maischter seets ,der Meister sagt'). Bei sozialer Distanz, Fremdheit oder respektbehaftetem Familienstatus (Mütter, Groß- und Schwiegermütter) gilt das Femininum bzw. Maskulinum (Sexus-GenusKongruenz). Für hochgeachtete Frauen existieren sogar gestärkte Sonderpronomen wie $d s c h o ̈ i$ ,sie', die sich für andere Feminina verbieten. Geschwister, Kinder und Freunde sind dagegen neutrumaffin, gerade bei der Pronominalisierung maskuliner und femininer Personenbezeichnungen (,mein Bruder $-\mathrm{es}^{`}$ ). Da die Dorfgemeinschaften klein und das Verhältnis unter ihren Mitgliedern eng ist, ist das Neutrum allgegenwärtig. Kontexte des Ihrzens und der Verwendung honorativer Anreden sind mittlerweile so selten, dass das Neutrum zum unmarkierten Genus avanciert ist und weiterhin zunimmt: „Das ganze Feld der Personen-Pronominalisierung ist im Begriff, dem Neutrum zuzufallen.“

\section{Literaturverzeichnis}

Braun, Friederike/Haig, Geoffrey (2010): „When are German ,girls“ feminine? How the semantics of age influences the grammar of gender agreement". In: Bieswanger, Markus/Motschenbacher, Heiko/Mühleisen, Susanne (eds.): Language in its socio-cultural context: New explorations in global, medial and gendered uses. Frankfurt, Peter Lang: 69-84.

Christen, Helen (1998): „Die Mutti oder das Mutti, die Rita oder das Rita? Über Besonderheiten der Genuszuweisung bei Personen- und Verwandtschaftsnamen in schweizerdeutschen Dialekten“. In: Schnyder, André. et al. (eds.): Ist mir getroumet mîn leben? Vom Träumen und vom Anderssein. Festschrift für Karl-Ernst Geith zum 65. Geburtstag. Göppingen, Kümmerle: 267-281. (= Göppinger Arbeiten zur Germanistik 632).

Corbett, Greville G. (1991): Gender. Cambridge: University Press.

Corbett, Greville G. (2006): Agreement. Cambridge: University Press. 